'Servicio de Anatomía Patológica, Hospital Clínico Universidad de Chile.

${ }^{2}$ Instituto Nacional del Tórax. Facultad de Medicina, Universidad de Chile. 3Unidad de Tórax, Centro de Imagenología, Hospital Clínico Universidad de Chile. aMédico Becado de Radiología.

Fuente de apoyo financiero: No.

Recibido el 26 de noviembre de 2013, aceptado el 10 de abril de 2014.

Correspondencia a:

Dr. Mauricio Salinas F. José Manuel Infante 717 , Providencia, Santiago. mrsf24@hotmail.com

\section{Microlitiasis alveolar pulmonar: caso clínico}

\author{
CRISTINA FERNÁNDEZ F. ${ }^{1}$, MAURICIO SALINAS F. ${ }^{2}$, JOSÉ ANDRÉS DE \\ GRAZIA K. ${ }^{3, a}$, JUAN CARLOS DÍAZ $\mathrm{P}^{3}$
}

\section{Pulmonary alveolar microlithiasis. Report of one case}

\begin{abstract}
Pulmonary alveolar microlithiasis is an extremely rare disease characterized by intra-alveolar accumulation of calcified spherical particles (called microliths), due to a mutation of the gene encoding a membrane transport protein of the alveolar surface. Most patients are asymptomatic at diagnosis. The course of the disease is slowly progressive, with development of pulmonary fibrosis and respiratory failure. The "sandstorm" pattern is the characteristic finding of this disease. We report a 39-year-old female presenting with progressive dyspnea. A chest $X$ ray showed ground-glass opacities and a high resolution CT scan showed numerous calcified lung micronodules. A surgical lung biopsy confirmed the diagnosis of pulmonary alveolar microlithiasis.

(Rev Med Chile 2014; 142: 656-661)

Key words: Lung diseases; Pulmonary alveolar microlithiasis; Tomography scanners, X-Ray computed.
\end{abstract}

$\mathrm{E}$ l enfrentamiento de las enfermedades pulmonares difusas constituye con frecuencia un desafío diagnóstico para el clínico, el radiólogo e incluso el patólogo dependiendo de la enfermedad específica y el tipo de biopsia lograda.

Más allá de la fibrosis pulmonar idiopática, la enfermedad pulmonar difusa primaria pulmonar por excelencia, existen innumerables entidades y condiciones de diagnóstico difícil y complejo, muchas de las cuales son enfermedades infrecuentes o derechamente enfermedades huérfanas y que por lo mismo no se plantean rutinariamente.

La microlitiasis alveolar pulmonar está en este último grupo y el caso presentado a continuación, que es el primero publicado en Chile de acuerdo a nuestra revisión, confirma que el enfrentamiento de las enfermedades pulmonares difusas requiere una aproximación detallista y crítica, por un grupo dedicado al tema. Sólo de esta forma, se obtendrá un diagnóstico preciso, tratamiento adecuado y un pronóstico real de la situación del enfermo.

\section{Caso clínico}

Paciente mujer de 39 años, de nacionalidad peruana, radicada en Chile desde hace 15 años, sin antecedentes mórbidos, no fumadora.

Consultó al médico con historia de varios meses de disnea progresiva, acentuada en el contexto de un cuadro respiratorio agudo febril que fue manejado en forma ambulatoria con antibióticos y corticoides. La respuesta al tratamiento fue parcial, con regresión del cuadro febril pero con persistencia de la disnea de esfuerzo, por lo que consultó en el Instituto Nacional del Tórax. En su primera evaluación la paciente presentaba una saturación por oximetría de pulso de $96 \%$ respirando aire ambiental, no existiendo hallazgos relevantes en el examen físico general o segmentario. Los exámenes de función pulmonar mostraron capacidad vital forzada (CVF) de $91 \%$, volumen espiratorio forzado en un segundo (VEF-1) de $99 \%$ y capacidad de difusión pulmonar (DLCO) de 62\%.

La radiografía de tórax (Figura 1) mostraba 
opacidad en vidrio esmerilado difusa, de predominio en las bases pulmonares, con zonas más densas que producen signo de la silueta con el corazón.

La tomografía computada (TC) de tórax de alta resolución (Figuras 2 y 3 ) mostró innumerables micronódulos pulmonares calcificados, de

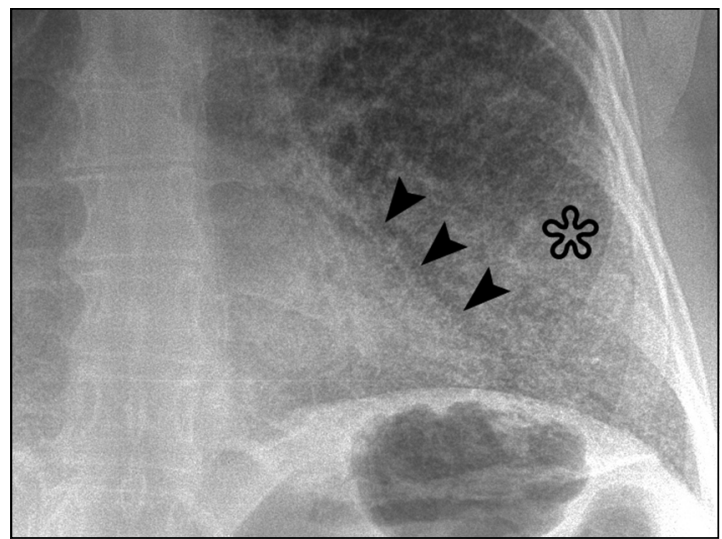

Figura 1. Radiografía de tórax PA, detalle de la base pulmonar izquierda. Se observa opacidad en vidrio esmerilado difusa, de predominio en la base pulmonar (asterisco), con zonas más densas que producen signo de la silueta con el corazón (cabezas de flecha). predominio subpleural, en mayor cantidad en los segmentos anteriores de los lóbulos superiores y posteriores de los lóbulos inferiores, asociados a engrosamiento de aspecto nodular del intersticio subpleural y peribroncovascular, junto con áreas de densidad en vidrio esmerilado mayores en las bases pulmonares.

Se interrogó dirigídamente sobre exposiciones ambientales u ocupacionales y se realizó estudio de laboratorio de enfermedades reumatológicas, todo lo cual resultó negativo. Los niveles de calcio, fósforo y albúmina plasmáticos fueron normales.

Se estudia con fibrobroncoscopia que no mostró alteraciones macroscópicas, con lavado bronco-alveolar sin hallazgos diagnósticos. El estudio microbiológico con cultivo corriente, baciloscopia y cultivo de Löwenstein-Jensen fueron negativos.

Finalmente, se realizó una biopsia pulmonar por videotoracoscopia. En el estudio macroscópico se observaron fragmentos de pulmón con pleura delgada y traslúcida, con parénquima pulmonar amarillo dorado de apariencia finamente granular. En el estudio microscópico (Figura 4) se reconoció pulmón con pleura y septos interlobulillares normales, de arquitectura conservada,
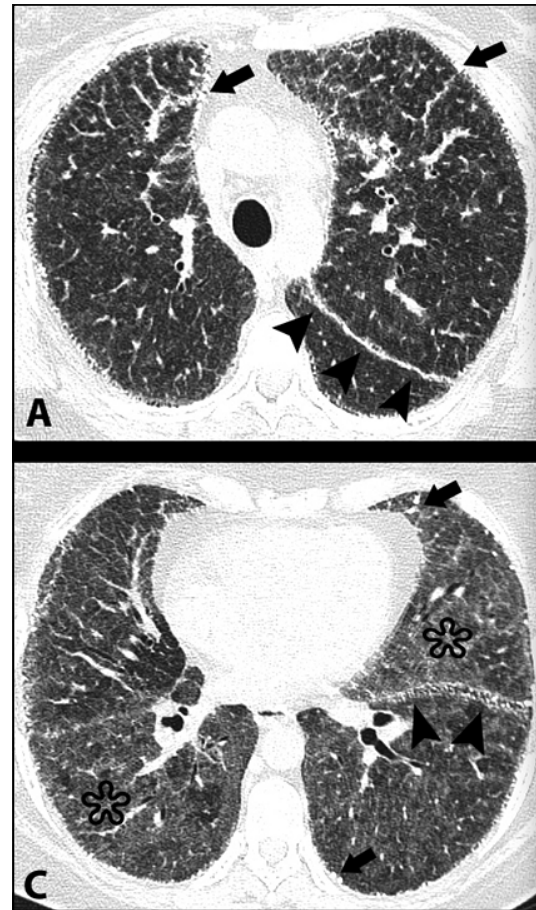

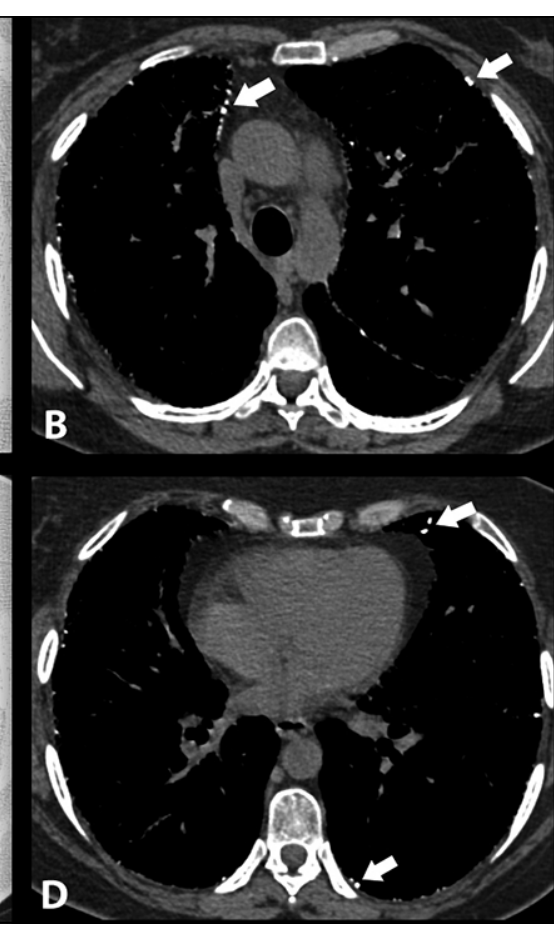

Figura 2. Cortes axiales de TC de tórax de alta resolución. Las imágenes $A$ y $B$ son a nivel de los lóbulos superiores, en ventana pulmonar y mediastínica respectivamente; las imágenes C y D son a un nivel más bajo, también en ventana pulmonar y mediastínica respectivamente. Se observan múltiples micronódulos pulmonares de predominio subpleural, los cuales en ventana mediastínica demuestran ser de densidad cálcica (flechas). Existe además engrosamiento de aspecto nodular del intersticio subpleural (cabezas de flecha) y áreas de densidad en vidrio esmerilado mayores en las bases (asteriscos). 

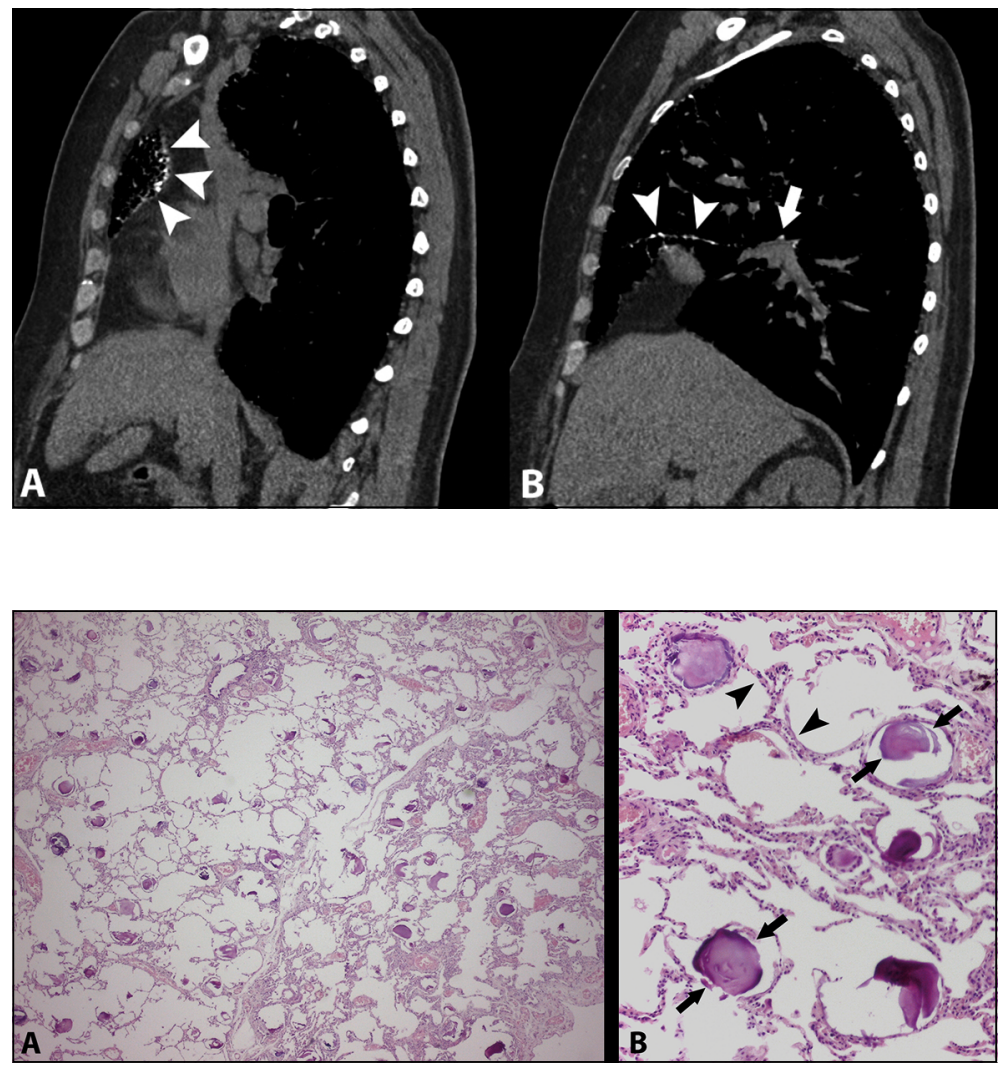

Figura 3. Reconstrucciones sagitales de TC de tórax de alta resolución en ventana mediastínica. Se observan múltiples micronódulos pulmonares calcificados de predominio subpleural adyacente a las cisuras y a la pleura parietal, asociados a engrosamiento del intersticio en esta misma localización (cabezas de flecha). Existen también micronódulos calcificados adyacentes al intersticio peribroncovascular (flecha).

Figura 4. Biopsia pulmonar, imágenes de la histología con tinción de Hematoxilina-Eosina, con aumento de $4 x$ (imagen A) y 40x (imagen B). Se observa ocupación parcial y difusa del espacio aéreo alveolar por calcificaciones calcáreas concéntricas, correspondientes a microlitos (flechas). El parénquima pulmonar presenta arquitectura conservada, con paredes alveolares delgadas (cabezas de flecha). con paredes alveolares delgadas, con ocupación parcial y difusa del espacio aéreo alveolar por calcificaciones calcáreas concéntricas.

Considerando el cuadro clínico, los hallazgos imagenológicos y el resultado de la biopsia el diagnóstico es microlitiasis alveolar pulmonar.

\section{Discusión}

La microlitiasis alveolar pulmonar es una enfermedad poco frecuente, con alrededor de 500 casos descritos en la literatura, la mayoría en Turquía e Italia ${ }^{1,2}$. Afecta a personas de cualquier edad, aunque el diagnóstico se realiza usualmente en la tercera o cuarta década de vida, sin predominancia según $\operatorname{sexo}^{1-3}$. Alrededor de dos tercios de los casos se presentan como casos esporádicos $y$ un tercio como casos con agregación familiar ${ }^{1,2}$.

La enfermedad se caracteriza por la acumulación intra-alveolar de partículas calcificadas esféricas (llamadas microlitos). Se debe a la mutación homocigota, de herencia autosómica recesiva, del gen SLC34A2 ubicado en el brazo corto del cromosoma 15 (4p15), el cual codifica para una proteína de membrana transportadora de fosfato dependiente de sodio, ubicada en la superficie apical de los neumocitos tipo II (transportador de fosfato dependiente de sodio tipo IIb) ${ }^{3-6}$. Cuando existe esta mutación, la función de la proteína transportadora se altera y se acumula fosfato derivado de la degradación de fosfolípidos del surfactante al interior de los alveolos. La acumulación de fosfato determina el depósito de calcio y la consiguiente formación de microlitos.

La mayoría de los pacientes son asintomáticos al momento del diagnóstico, siendo pesquisada la enfermedad en forma incidental en exámenes de imágenes ${ }^{3}$. En los casos avanzados, los síntomas principales son disnea progresiva y tos $\mathrm{seca}^{1-4}$. El curso de la enfermedad es lentamente progresivo, con desarrollo de fibrosis pulmonar, insuficiencia respiratoria, cor pulmonale y posteriormente la muerte ${ }^{1-4,7}$. La sobrevida promedio de los pacien- 
tes desde el diagnóstico es de 10 a 15 años ${ }^{8}$. Los pacientes fumadores presentan una progresión más rápida ${ }^{3}$.

Las pruebas de función pulmonar en un comienzo son normales, presentando en etapas avanzadas un patrón restrictivo, disminución de la capacidad de difusión pulmonar y finalmente hipoxemia ${ }^{3}$. El estudio de laboratorio de metabolismo del calcio es normal ${ }^{3}$. Estudios recientes han demostrado elevación de los niveles plasmáticos de las proteínas A y D del surfactante, en magnitud proporcional al grado de deterioro de la función pulmonar, por lo que podrían servir como marcadores de progresión de la enfermedad ${ }^{9}$.

El transportador de fosfato dependiente de sodio tipo IIb se expresa en menor medida en órganos distintos al pulmón, como el riñón, vesículas seminales, epidídimo, testículo y vesícula biliar, lo que explica la existencia de manifestaciones extrapulmonares asociadas, como nefrocalcinosis, nefrolitiasis, azoospermia obstructiva, calcificaciones testiculares y colelitiasis ${ }^{3}$.

En la radiografía de tórax la enfermedad se caracteriza por micronódulos pulmonares calcificados, de distribución bilateral, con predominio en las bases, con aspecto en "tormenta de arena"1-3,10. Puede existir signo de la silueta con el corazón o el diafragma debido a una gran cantidad y densidad de microlitos ${ }^{3,10,11}$. Otro hallazgo menos frecuente es la presencia de una fina banda radiolúcida entre el parénquima pulmonar calcificado y las costillas o el mediastino ("pleura negra"), secundaria a

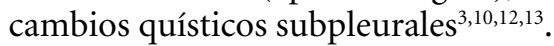

La TC de tórax de alta resolución es el examen de imágenes de elección. El hallazgo característico es la presencia de innumerables microlitos bilaterales, con predominio en los segmentos posteriores de los lóbulos inferiores y los segmentos anteriores de los lóbulos superiores ${ }^{2,3,10-13}$. En los lóbulos superiores pueden visualizarse áreas de calcificación confluente ${ }^{10-12}$. Existen altas concentraciones de microlitos en la periferia de los lobulillos pulmonares secundarios, los cuales son los responsables del aspecto engrosado y micronodular de los septos interlobulillares ${ }^{10-12}$. Pueden verse también microlitos adyacentes al intersticio peribroncovascular y subpleural, resultando en un aspecto engrosado y micronodular de estas estructuras ${ }^{10}$. El acúmulo de microlitos de un diámetro menor a $1 \mathrm{~mm}$ determina la formación de densidades en vidrio esmerilado e incluso focos de condensación densos, los cuales con configuración de ventana mediastínica (ancho de ventana 300-500 UH, nivel de ventana $40 \mathrm{UH}$ ) pueden ser observados como múltiples calcificaciones discretas $^{10,11}$. El engrosamiento de los septos interlobulillares asociado a las densidades en vidrio esmerilado pueden crear un aspecto en "crazy paving", similar al de la proteinosis alveolar ${ }^{3,10,14}$. Numerosos quistes subpleurales de entre 5 y 10 $\mathrm{mm}$, de pared fina, son otro hallazgo frecuente $\mathrm{y}$ son los determinantes de la fina banda radiolúcida subpleural visible en la radiografía de tórax ("pleura negra") 3,10,12,13. Es frecuente una disociación entre la clínica y los hallazgos radiológicos, con pacientes asintomáticos u oligosintomáticos que presentan importantes alteraciones radiológicas $^{1-3}$. El conjunto de hallazgos tomográficos es altamente específico de la enfermedad ${ }^{1-3}$.

Dentro de los diagnósticos diferenciales debe considerarse la sarcoidosis, silicosis, hemosiderosis pulmonar idiopática, amiloidosis, linfangitis carcinomatosa, tuberculosis miliar, calcificaciones pulmonares metastásicas asociadas a insuficiencia renal crónica y proteinosis alveolar ${ }^{3,10,11,15-17}$.

El estudio histológico demuestra microlitos en los espacios alveolares, los cuales se visualizan como partículas esféricas, de entre 0,1 y $2,8 \mathrm{~mm}$ de diámetro, con capas calcáreas concéntricas, positivas en la tinción periodic acid-Schiff $(P A S)^{3,8,18}$. $\mathrm{Su}$ composición es de fosfato de calcio e hidroxiapatita y carboxiapatita de calcio ${ }^{18}$. Su aspecto es distinto al de las calcificaciones metastásicas y distróficas, las cuales se localizan en los compartimientos intersticial y vascular ${ }^{17}$. El depósito de microlitos comienza en los lóbulos inferiores y posteriormente progresa hacia los ápices pulmonares. En etapas iniciales los septos interlobulillares se encuentran intactos y el intercambio gaseoso es normal. A medida que los microlitos crecen al interior de los alveolos, contactan las paredes alveolares, ejerciendo presión e irritación, lo cual determina la presencia de un proceso inflamatorio crónico en el espacio intersticial y el progresivo reemplazo de las paredes alveolares por tejido fibroso ${ }^{2,3,18}$.

En la actualidad no existe un tratamiento específico para esta enfermedad ${ }^{3}$. Se ha intentado el uso de corticoides sistémicos, lavados broncoalveolares terapéuticos y bifosfonatos, los dos primeros sin resultados favorables ${ }^{4}$ y el último con resultados disímiles ${ }^{19,20}$. Un grupo turco 
ha publicado casos de tratamiento exitoso con etidronato $^{20}$, un bifosfonato no disponible en nuestro país. Muchos pacientes requieren uso de $\mathrm{O}_{2}$ domiciliario a medida que la enfermedad progresa. En casos avanzados el trasplante pulmonar es la única opción ${ }^{3,21}$. No se dispone actualmente de series de casos en la literatura que evalúen la sobrevida de estos pacientes y la tasa de recurrencia de la enfermedad luego del trasplante.

El caso presentado es característico de esta rara enfermedad, con una paciente en la cuarta década de la vida, con cuadro clínico oligosintomático y TC de tórax con floridas manifestaciones, lo cual pone de manifiesto la disociación clínico-radiológica característica de esta enfermedad. El estudio histológico confirma el diagnóstico al demostrar ocupación difusa parcial del espacio aéreo alveolar por calcificaciones concéntricas correspondientes a microlitos.

La paciente inició tratamiento con alendronato $70 \mathrm{mg}$ semanal luego del diagnóstico, suspendiendo corticoides, luego de lo cual tuvo mejoría clínica y de función pulmonar; la CVF inicial de $91 \%$ subió a $96 \%$ y la DLCO de $62 \%$ subió a $81 \%$ en evaluaciones realizadas alrededor de 6 meses luego de iniciado el tratamiento. Las imágenes de TC no se han modificado.

\section{Referencias}

1. Castellana G, Lamorgese V. Pulmonary alveolar microlithiasis. World cases and review of the literature. Respiration 2003; 70 (5): 549-55.

2. Mariotta S, Ricci A, Papale M, De Clementi F, Sposato B, Guidi L, et al. Pulmonary alveolar microlithiasis: report on 576 cases published in the literature. Sarcoidosis Vasc Diffuse Lung Dis 2004; 21 (3): 173-81.

3. Ferreira Francisco FA, Pereira e Silva JL, Hochhegger B, Zanetti G, Marchiori E. Pulmonary alveolar microlithiasis. State-of-the-art review. Respir Med 2013; 107 (1): 1-9.

4. Tachibana T, Hagiwara K, Johkoh T. Pulmonary alveolar microlithiasis: review and management. Curr Opin Pulm Med 2009; 15 (5): 486-90

5. Huqun Izumi S, Miyazawa H, Ishii K, Uchiyama B, Ishida T, Tanaka S, et al. Mutations in the SLC34A2 gene are associated with pulmonary alveolar microlithiasis. Am J Respir Crit Care Med 2007; 175 (3): 263-8.

6. Corut A, Senyigit A, Ugur SA, Altin S, Ozcelik U, Calisir $\mathrm{H}$, et al. Mutations in SLC34A2 cause pulmonary alveolar microlithiasis and are possibly associated with testicular microlithiasis. Am J Hum Genet 2006; 79 (4): 650-6.

7. Terada T. Pulmonary alveolar microlithiasis with cor pulmonale: an autopsy case demonstrating a marked decrease in pulmonary vascular beds. Respir Med 2009; 103 (11): 1768-71.

8. Lauta VM. Pulmonary alveolar microlithiasis: an overview of clinical and pathological features together with possible therapies. Respir Med 2003; 97 (10): 1081-5.

9. Takahashi H, Chiba H, Shiratori M, Tachibana T, Abe S. Elevated serum surfactant protein A and D in pulmonary alveolar microlithiasis. Respirology 2006; 11 (3): 330-3.

10. Siddiqui NA, Fuhrman CR. Best cases from the AFIP: Pulmonary alveolar microlithiasis. Radiographics 2011; 31 (2): 585-90.

11. Deniz O, Ors F, Tozkoparan E, Ozcan A, Gumus S, Bozlar $\mathrm{U}$, et al. High resolution computed tomographic features of pulmonary alveolar microlithiasis. Eur J Radiol 2005; 55 (3): 452-60.

12. Korn MA, Schurawitzki H, Klepetko W, Burghuber OC. Pulmonary alveolar microlithiasis: findings on highresolution CT. AJR Am J Roentgenol 1992; 158 (5): 981-2.

13. Cluzel P, Grenier P, Bernadac P, Laurent F, Picard JD. Pulmonary alveolar microlithiasis: CT findings. J Comput Assist Tomogr 1991; 15 (6): 938-42.

14. Gasparetto EL, Tazoniero P, Escuissato DL, Marchiori E, Frare E Silva RL, et al. Pulmonary alveolar microlithiasis presenting with crazy-paving pattern on high resolution CT. Br J Radiol 2004; 77 (923): 974-6.

15. Saputo V, Zocchi M, Mancosu M, Bonaldi U, Croce P. Pulmonary alveolar microlithiasis. A case report with a discussion of differential diagnosis. Helv Paediatr Acta 1979; 34 (3): 245-55.

16. Weinstein DS. Pulmonary sarcoidosis: calcified micronodular pattern simulating pulmonary alveolar microlithiasis. J Thorac Imaging 1999; 14 (3): 218-20.

17. Chan ED, Morales DV, Welsh CH, McDermott MT, Schwarz MI. Calcium deposition with or without bone formation in the lung. Am J Respir Crit Care Med 2002; 165 (12): 1654-69.

18. Barnard NJ, Crocker PR, Blainey AD, Davies RJ, Ell SR, Levison DA. Pulmonary alveolar microlithiasis. A new analytical approach. Histopathology 1987; 11 (6): 63945.

19. Ozcelik U, Yalcin E, Ariyurek M, Ersoz DD, Cinel G, Gulhan $\mathrm{B}$, et al. Long-term results of disodium etidronate treatment in pulmonary alveolar microlithiasis. Pediatr Pulmonol 2010; 45 (5): 514-7. 
Microlitiasis Alveolar Pulmonar - C. Fernández et al

20. Jankovic S, Pavlov N, Ivkosic A, Erceg I, Glavina-Durdov $\mathrm{M}$, Tocilj J, et al. Pulmonary alveolar microlithiasis in childhood: clinical and radiological follow-up. Pediatr Pulmonol 2002; 34 (5): 384-7.
21. Stamatis G, Zerkowski HR, Doetsch N, Greschuchna D, Konietzko N, Reidemeister JC. Sequential bilateral lung transplantation for pulmonary alveolar microlithiasis. Ann Thorac Surg 1993; 56 (4): 972-5. 